\title{
Predictors of pericardial effusion after orthotopic heart transplantation
}

\author{
Jacquelyn A. Quin, MD \\ M. Peter Tauriainen, MD \\ Lynne M. Huber, RN \\ Donald D. McIntire, PhD \\ Patricia A. Kaiser, RN \\ W. Steves Ring, MD \\ Michael E. Jessen, MD
}

From the Department of Cardiovascular and Thoracic Surgery, University of Texas Southwestern Medical Center at Dallas, Tex.

Received for publication Sept 13, 2001; revisions requested Jan 3, 2002; revisions received Jan 23, 2002; accepted for publication Feb 7, 2002.

Address for reprints: Michael E. Jessen, MD, Department of Cardiovascular and Thoracic Surgery, University of Texas Southwestern Medical Center, 5323 Harry Hines Blvd, Dallas, TX 75390-8879 (E-mail: michael.jessen@utsouthwestern .edu).

J Thorac Cardiovasc Surg 2002;124:979-83

Copyright $(\odot 2002$ by The American Association for Thoracic Surgery

0022-5223/2002 \$35.00+0 $\quad \mathbf{1 2 / 1 / 1 2 4 3 8 7}$

doi: $10.1067 / \mathrm{mtc} .2002 .124387$
Objectives: Pericardial effusion occurs frequently after orthotopic heart transplantation, but the causes of this complication have not been well described. This study was designed to identify factors predisposing toward the development of significant postoperative pericardial effusions in a large, single-institution population of orthotopic heart transplant recipients.

Methods: A retrospective review of more than 90 preoperative, intraoperative, and postoperative variables was conducted for 241 patients undergoing orthotopic heart transplantation from September 1988 to December 1999. Patients who had significant postoperative pericardial effusions develop were identified from postoperative echocardiograms by standard criteria. Factors associated with the development of significant pericardial effusions were determined by multivariate logistic regression analysis.

Results: Echocardiographic data were available for 203 of 241 transplant recipients. Forty-two patients (21\%) had significant effusions develop. According to multivariate analysis, pericardial effusions were less likely to occur in recipients with a history of previous cardiac surgery (odds ratio 0.13, 95\% confidence interval 0.05-0.36, $P<.0001$ ) and with greater weight (odds ratio $0.96,95 \%$ confidence interval $0.94-0.99, P<.0048$ ). Pericardial effusions were more likely to occur in patients who had received aminocaproic acid during the operation (odds ratio 5.92, 95\% confidence interval 2.23-15.72, $P<.0008$ ). Patient survival and hospital length of stay did not differ between patients with and without postoperative pericardial effusions.

Conclusions: Postoperative pericardial effusions develop in approximately $20 \%$ of patients undergoing orthotopic cardiac transplantation. On the basis of the risk factors identified in this study, prevention may prove difficult, although avoidance of the intraoperative use of aminocaproic acid may be helpful.

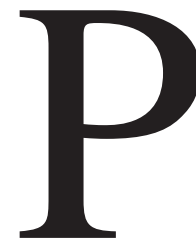

ericardial effusion occurs frequently after all forms of cardiac surgery, ${ }^{1}$ and its appearance has been associated with postoperative bleeding, ${ }^{2}$ postpericardiotomy syndrome,${ }^{3}$ and postoperative anticoagulation. ${ }^{4}$ Effusions are frequently detected after orthotopic cardiac transplantation, but the factors associated with the development of pericardial effusion in this setting may differ from those in other situations. Moderate or large pericardial effusions have been reported to occur more frequently among transplant recipients with no previous cardiac surgery, $, 5,6$ those with a greater recipient-donor weight difference, ${ }^{6}$ and those who receive cyclosporine (INN: ciclosporin) as part of the immunosuppressive protocol. ${ }^{7}$ The relationship 


\section{TABLE 1. Variables included in the analysis}

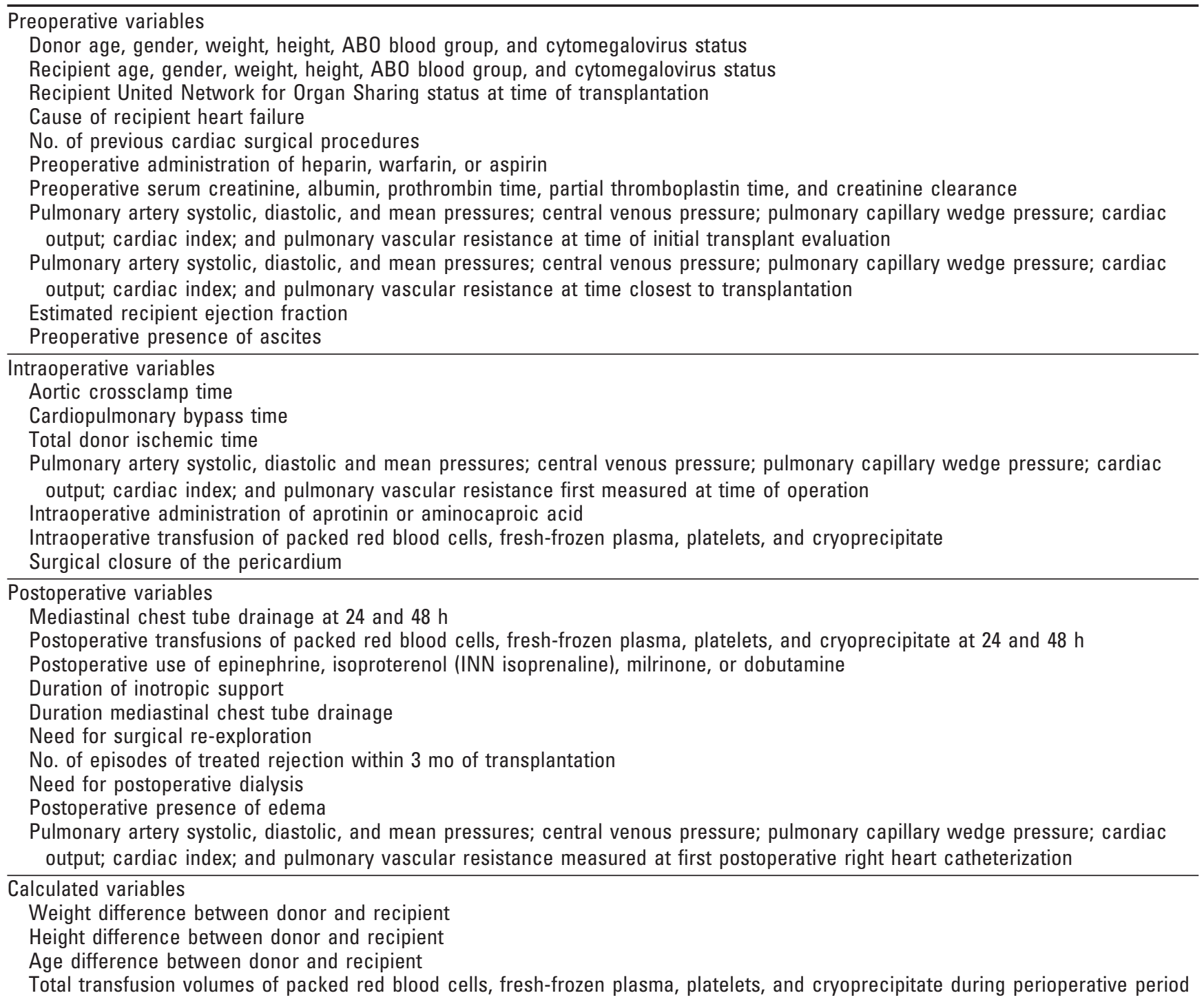

between episodes of acute rejection and the genesis of pericardial effusion is controversial. Ciliberto and associates $^{5}$ and Valantine and colleagues ${ }^{8}$ have reported positive associations between acute rejection and posttransplantation effusion. However, a larger multivariate analysis by Hauptman and colleagues ${ }^{6}$ did not discern any relationship between these events.

Pericardial effusions tend to occur within the first 3 months after transplantation, and late development is uncommon. ${ }^{8}$ Their appearance may mandate surgical or catheter drainage, but the effect of effusions on survival or other outcome measures has not been well established. This study was undertaken to identify characteristics of the donor, the recipient, the operative procedure, and the early postoperative course that predict the development of significant peri- cardial effusion after orthotopic cardiac transplantation. The study also examined the influence of pericardial effusions on common posttransplantation outcome measures.

\section{Methods}

A retrospective review was conducted of 241 consecutive patients undergoing orthotopic heart transplantation at St Paul Medical Center in Dallas from September 1988 (when the program began) to December 1999. Data collected included preoperative donor and recipient demographic and clinical information, intraoperative variables related to the conduct of the transplant operation, and postoperative variables describing the hemodynamic course, bleeding and transfusion requirements, and selected postoperative complications. A complete list of variables collected and analyzed is presented in Table 1. 
Patients in whom postoperative pericardial effusions developed were identified from echocardiograms performed within the first 3 months after transplantation. Effusions occurring later were not considered in this study. Pericardial effusions when present were graded according to the guidelines established by Martin and coworkers. ${ }^{9}$ Small effusions were characterized as fluid collections limited to the atrioventricular groove. Fluid collections that were also distributed laterally, apically, or anteriorly were characterized as moderate. Large fluid collections extended further posteromedially or circumferentially. On the basis of these findings, patients were placed into two groups according to effusion size: those with moderate or large effusions were considered to have significant pericardial effusion, whereas those with small or undetectable effusions were deemed to have no significant pericardial effusion. Effusions that necessitated operative or catheter drainage procedures were also noted.

Outcome data, including recipient survival, length of postoperative hospital stay, and rejection episodes, were also recorded for all patients. The incidence of posttransplantation graft rejection was assessed by review of all surveillance biopsies done for each patient within the first 3 months after transplantation. Rejection episodes were recorded if biopsy specimens demonstrated histologic changes of grade 2 or higher according to the International Society for Heart \& Lung Transplantation guidelines ${ }^{10}$ and patients received treatment for rejection.

\section{Statistical Analysis}

Data were analyzed with commercially-available software (SAS Institute, Inc, Cary, NC). A univariate analysis of all variables listed in Table 1 was performed initially to assess for differences between groups with and without significant pericardial effusion. A $\chi^{2}$ analysis was used for discrete variables, with $P<.05$ according to 2-tailed Fisher exact test used to select factors with potential significance. For continuous variables, the Student $t$ test with the Welch approximation for unequal variances was used to compare the two groups. Group differences were also considered potentially significant at $P<.05$.

To account for a lack of independence among the variables, additional testing was used. Variables with the potential for significant difference between groups according to univariate analysis were entered as candidate variables in a mulitvariate stepwise logistic regression analysis. For each element remaining in the multivariate model, a parameter estimate was calculated from which a $P$ value, odds ratio (OR), and $95 \%$ confidence interval (CI) for the variable were derived.

To examine patient survival and freedom from rejection, estimates based on the Kaplan-Meier method were created and compared statistically with a log-rank test. Data are expressed as mean \pm SD except in the case of survival data, which are reported as mean \pm SEM.

\section{Results}

Between September 1988 and December 1999, a total of 241 patients underwent orthotopic heart transplantation. Two hundred three patients had documented echocardiograms within the first 3 postoperative months, and these patients form the study population. Forty-two patients had
TABLE 2. Variables found to differ significantly between patients with and without pericardial effusion after orthotopic heart transplantation, as determined by univariate analysis

\begin{tabular}{|c|c|c|c|}
\hline Variable & $\begin{array}{c}\text { No } \\
\text { significant } \\
\text { effusion }\end{array}$ & $\begin{array}{l}\text { Significant } \\
\text { effusion }\end{array}$ & $\begin{array}{c}P \\
\text { value }\end{array}$ \\
\hline Recipient age (y) & $54.3 \pm 0.8$ & $49.2 \pm 1.9$ & $<.01$ \\
\hline $\begin{array}{l}\text { Preoperative central venous } \\
\text { pressure }(\mathrm{mm} \mathrm{Hg})\end{array}$ & $15.5 \pm 0.6$ & $19.7 \pm 1.8$ & $<.04$ \\
\hline $\begin{array}{l}\text { Recipient diagnosis of idiopathic } \\
\text { dilated cardiomyopathy }\end{array}$ & $24 \%$ & $50 \%$ & $<.001$ \\
\hline Previous cardiac surgery & $54 \%$ & $12 \%$ & $<.001$ \\
\hline Female donor heart & $30 \%$ & $52 \%$ & $<.01$ \\
\hline Recipient weight (kg) & $75.6 \pm 1.2$ & $69.8 \pm 2.0$ & $<.03$ \\
\hline $\begin{array}{l}\text { Intraoperative use of } \\
\text { aminocaproic acid }\end{array}$ & $7 \%$ & $33 \%$ & $<.001$ \\
\hline Postoperative pedal edema & $55 \%$ & $32 \%$ & $<.01$ \\
\hline
\end{tabular}

Data are presented as mean \pm SEM for continuous variables and as percentages of patients for discrete variables. Comparisons are by Student $t$ test for continuous variables and by Fisher exact test for discrete variables.

significant pericardial effusion develop (13 large and 29 moderate effusions) according to the criteria described previously, and 19 of these patients required drainage. When drainage was required, it was achieved by placement of a subxiphoid pericardiostomy tube. In 2 cases the effusion recurred, ultimately necessitating a pericardial window through a limited thoracotomy. None of the patients underwent prophylactic drainage of the pericardium into the pleural space at the time of the initial operation.

Variables found to correlate with significant pericardial effusion according to the univariate analysis are listed in Table 2. Patients with significant pericardial effusion were less likely to have had previous cardiac surgery and more likely to be undergoing transplantation for idiopathic dilated cardiomyopathy, to be younger, to be lighter, and to have higher central venous pressure at time of transplant evaluation. The use of hearts from female donors was associated with significant effusion, as was intraoperative administration of the antifibrinolytic agent aminocaproic acid (Amicar). Patients with significant pericardial effusion were found to have a lower incidence of postoperative pedal edema.

Of the candidate variables found by univariate analysis, three emerged as possible predictors of significant pericardial effusion according to the multivariate logistic regression analysis (Table 3). Previous cardiac surgery was the greatest deterrent to the development of pericardial effusion (OR 0.13, 95\% CI 0.05-0.36, $P<.0002$ ). The use of aminocaproic acid increased the relative risk of pericardial effusion nearly 6-fold. A greater recipient weight at the time 
TABLE 3. Variables found to differ significantly between patients with and without pericardial effusion after orthotopic heart transplantation, as determined by multivariate logistic regression analysis

\begin{tabular}{lclc}
\hline Variable & OR & \multicolumn{1}{c}{$\mathbf{9 5 \%} \mathbf{C l}$} & $\boldsymbol{P}$ value \\
\hline $\begin{array}{l}\text { Previous cardiac procedure } \\
\text { Intraoperative use of }\end{array}$ & 0.13 & $0.05-0.36$ & .0001 \\
$\quad$ aminocaproic acid & 5.92 & $2.23-15.72$ & .0008 \\
Greater recipient weight & 0.96 & $0.94-0.99$ & .0048 \\
\hline
\end{tabular}

of transplantation emerged as slightly protective against the development of pericardial effusion.

No association was found between rejection and the development of pericardial effusions. Forty-five patients required treatment for acute graft rejection within the first 3 months: $11(26 \%)$ of 42 patients with pericardial effusions and $34(21 \%)$ of 161 patients without pericardial effusions $(P=.48)$. The 5-year freedoms from rejection of patients with and without pericardial effusions were $73 \% \pm 7 \%$ and $77 \% \pm 3 \%$, respectively $(P=.66)$. No correlation was seen between the development of significant effusions and either the duration or quantity of mediastinal chest tube drainage or the quantity of blood and blood products administered in the first 48 hours after transplantation.

The development of a postoperative pericardial effusion did not appear to be related to any common outcome measures in this population. Actuarial survival calculated by the Kaplan-Meier method was not different between transplant recipients with and without significant postoperative effusions. The 5-year survivals of patients with and without pericardial effusions were $92 \% \pm 4 \%$ and $83 \% \pm 3 \%$, respectively $(P=.15)$. Postoperative hospital stays were $19 \pm 3$ days and $18 \pm 1$ days among patients with and without pericardial effusions, respectively $(P=.66)$.

\section{Discussion}

Depending on the definition, postoperative pericardial effusions may be found in as many as $70 \%$ of patients undergoing cardiac surgery. Most of these effusions are small and asymptomatic, and they generally resolve within 2 weeks of surgery, but some persist for several months and may even rarely cause tamponade. ${ }^{11}$ The incidence of pericardial effusions among patients undergoing orthotopic cardiac transplantation is similar to that associated with other open cardiac procedures. Again, definitions of significant effusions vary, but in this series of 203 patients, significant (moderate and large) pericardial effusions occurred in 21\%, an incidence similar to the reports of others. ${ }^{12}$ Although no patients with significant effusions had tamponade develop, nearly half of these patients $(n=19 / 42)$ did undergo a drainage procedure.

According to univariate analysis, recipients with dilated cardiomyopathy, no previous cardiac surgery, and younger age were more likely to have significant pericardial effusions develop. Other groups have reported associations with dilated cardiomyopathy ${ }^{12}$ and the absence of previous cardiac surgery. ${ }^{5,6}$ These variables are related, because patients receiving transplants for dilated cardiomyopathy are significantly younger and significantly less likely to have undergone previous cardiac surgical procedures. It is therefore not surprising that only one of these variables (the absence of previous cardiac surgery) emerged as an independent predictor of pericardial effusions in the multivariate analysis.

Although previous studies have suggested that the development of acute rejection may contribute to the development of significant pericardial effusions, ${ }^{5,8}$ no such association was identified in this study. However the incidence of early acute rejection in this cohort was relatively low (45 of 203 patients), which may have influenced our ability to detect an association. The use of cyclosporine, cited by others as a possible cause of pericardial effusions, ${ }^{7}$ could not be analyzed because all transplant recipients in this study were treated with this drug after transplantation.

A statistical association between recipient weight and the development of pericardial effusions was found, with heavier recipients slightly protected from this complication. This association was found only for absolute recipient weight and not for the donor-recipient weight difference ( $P=.17$ by univariate analysis), as has been reported by others. ${ }^{6}$ The reason for this finding was not determined by this study, but one might speculate that a larger recipient would have a greater pericardial surface over which postoperative pericardial fluid could be distributed or absorbed. This would be difficult to ascertain, because cardiac dimensions are typically grossly enlarged before transplantation, and body weight may not be a precise surrogate for heart size in patients with heart failure. Direct measurements of donor and recipient heart sizes with quantitative imaging may provide more meaningful comparative data.

Use of the antifibrinolytic agent aminocaproic acid (administered intravenously in a dose of $10 \mathrm{~g}$ after heparinization plus $5 \mathrm{~g}$ added to the cardiopulmonary bypass circuit) was found to be associated with a dramatically increased incidence of postoperative pericardial effusion, a finding not previously reported. Agents such as aminocaproic acid and aprotinin are used in the perioperative setting to reduce postoperative bleeding. ${ }^{13,14}$ They are used more frequently for patients who have had previous cardiac surgery, because of the increased risk for bleeding in this group. However, aminocaproic acid use was identified as a predictor of pericardial effusions independent of reoperative status according to the multivariate analysis, whereas no association with aprotinin was observed. It is possible that the administration of an antifibrinolytic agent may contribute to retention of clots within the pericardial sac. Subsequent clot 
lysis could encourage fluid inspissation, leading to an effusion. However, it is noteworthy that the amount of mediastinal chest drainage, duration of chest tube drainage, and the quantities of blood products administered after transplantation were not found to correlate with the development of pericardial effusions. Also, the amount of mediastinal blood loss within the first 48 postoperative hours was not different between patients who received aminocaproic acid $(1500 \pm 210 \mathrm{~mL})$, aprotinin $(1673 \pm 186 \mathrm{~mL})$, or neither $(1483 \pm 100 \mathrm{~mL})$.

This study has several limitations. It is retrospective in design, and some data could not be obtained because of the unavailability of records. Harvesting and implantation procedures were performed by several surgeons, and there may have been subtle differences in operative technique that could have influenced outcomes. In addition, there may have been evolutionary changes in management protocols or patient selection criteria during the 12-year study interval that were not accounted for in this analysis. Antifibrinolytic agents were administered according to the judgment of the surgeon, anesthesiologist, and perfusionist at the time of surgery rather than according to a predetermined protocol. Finally, echocardiographic data may be somewhat subjective, and other definitions of pericardial effusions may be more appropriate. We applied the echocardiographic criteria defined by Martin and coworkers, ${ }^{9}$ because these guidelines have been used in other reports examining pericardial effusions in transplantation. When the analysis was repeated with a significant pericardial effusion defined as one necessitating surgical or catheter drainage $(n=19)$, multivariate analysis identified a diagnosis of dilated cardiomyopathy (OR 8.03, 95\% CI 2.61-24.66, $P<.0001$ ) and use of aminocaproic acid (OR 5.44, 95\% CI 1.76-16.87, $P<$ $.0019)$ as significant predictors.

As with any retrospective study, there are limitations to the statistical analysis and its interpretation. The number of patients in whom pericardial effusions developed totaled only 42 , and the number of predictors initially screened by univariate techniques exceeded this value. Even the entry of 8 candidate variables into the multivariate analysis may risk overfitting with this number of outcomes. ${ }^{15}$ Continuous variables such as recipient weight are modeled with the assumption that the risk for the outcome is linear across the range of weights included. This analysis does not account for the possibility that risk is not linear (as might be seen if the risk for pericardial effusion increases for very low weights and then levels off at higher weights). Finally, the meaning of the significant effects observed in this or any other retrospective study must be interpreted with caution. The variables identified as predictors may represent surrogates for other conditions that more directly affect the outcome.

To our knowledge, this is the largest study evaluating factors contributing to the development of significant pericardial effusions after orthotopic cardiac transplantation.
Previous cardiac procedures and greater recipient weight appear protective, whereas the use of aminocaproic acid appears to increase the risk of this complication. An association with acute rejection was not identified. On the basis of the currently identified factors, the development of pericardial effusions may be difficult to prevent, although avoiding the use of aminocaproic acid should be considered. The development of pericardial effusion does not appear to prolong hospital stay or affect survival. However, the presence of one or more risk factors in the patient undergoing orthotopic heart transplantation should lead to heightened awareness of this potential complication.

\section{References}

1. Weitzman LB, Tinker WP, Kronzan I, Cohen ML, Glassman E, Spencer FC. The incidence and natural history of pericardial effusion after cardiac surgery — an echocardiographic study. Circulation. 1984; 69:506-11.

2. Stevenson LW, Child JS, Laks H, Kern L. Incidence and significance of early pericardial effusion after cardiac surgery. Am J Cardiol. 1984;54:848-51.

3. Ofori-Krakye SK, Tyberg TI, Geha AS, Hammond GL, Cohen LS, Langou RA. Late cardiac tamponade after open heart surgery: incidence, role of anticoagulants in its pathogenesis and its relationship to the postpericardiotomy syndrome. Circulation. 1981;63:1323-8.

4. Borkon AM, Schaff HV, Gardner TJ, Merrill WH, Brawley RK, Donahoo JS, et al. Diagnosis and management of postoperative pericardial effusions and late cardiac tamponade following open-heart surgery. Ann Thorac Surg. 1981;31:512-9.

5. Ciliberto GR, Anjos MC, Gronda E, Bonacina E, Danzi G, Colombo $\mathrm{P}$, et al. Significance of pericardial effusion after heart transplantation. Am J Cardiol. 1995;76:297-300.

6. Hauptman PJ, Couper GS, Aranki SF, Kartashov A, Mudge GH Jr, Loh E. Pericardial effusions after cardiac transplantation. J Am Coll Cardiol. 1994;23:1625-9.

7. Hastillo A, Thompson JA, Lower RR, Szentpetery S, Hess ML. Cyclosporine-induced pericardial effusion after cardiac transplantation. Am J Cardiol. 1987;59:1220-2.

8. Valantine HA, Hunt SA, Gibbons R, Billingham ME, Stinson EB, Popp RL. Increasing pericardial effusion in cardiac transplant recipients. Circulation. 1989;79:603-9.

9. Martin RP, Rakowski H, French J, Popp RL. Localization of pericardial effusion with wide angle phased array echocardiography. Am J Cardiol. 1978;42:904-12.

10. Billingham ME, Cary NR, Hammond ME, Kemnitz J, Marboe C, McCallister HA, et al. A working formulation for the standardization of nomenclature in the diagnosis of heart and lung rejection: Heart Rejection Study Group. The International Society for Heart Transplantation. J Heart Transplant. 1990;9:587-93.

11. D'Cruz IA, Overton DH, Pai GM. Pericardial complications of cardiac surgery: emphasis on the diagnostic role of echocardiography. J Card Surg. 1992;7:257-68

12. Vandenberg BF, Mohanty PK, Craddock KJ, Barnhart G, Hanrahan J, Szentpetery $\mathrm{S}$, et al. Clinical significance of pericardial effusion after heart transplantation. J Heart Transplant. 1988;7:128-34.

13. Vander Salm TJ, Kaur S, Lancey RA, Okike ON, Pezzella AT, Stahl $\mathrm{RF}$, et al. Reduction of bleeding after heart operations through the prophylactic use of $\epsilon$-aminocaproic acid. J Thorac Cardiovasc Surg. 1996;112:1098-107.

14. Prendergast TW, Furukawa S, Beyer AJ, Eisen HJ, McClurken JB, Jeevanandam V. Defining the role of aprotinin in heart transplantation. Ann Thorac Surg. 1996;62:670-4.

15. Harrell FE, Lee KL, Mark DB. Multivariable prognostic models: issues in developing models, evaluating assumptions and adequacy, and measuring and reducing errors. Stat Med. 1996;15:361-87. 